\title{
Hydrodynamic interactions in long chain polymers: Application of the Chebyshev polynomial approximation in stochastic simulations
}

\author{
Richard M. Jendrejack, Michael D. Graham, and Juan J. de Pablo \\ Department of Chemical Engineering and Rheology Research Center, University of Wisconsin-Madison, \\ Madison, Wisconsin 53706-1691
}

(Received 23 February 2000; accepted 18 May 2000)

\begin{abstract}
We have simulated Brownian bead-spring chains of up to 125 units with fluctuating hydrodynamic and excluded volume interactions using the Chebyshev polynomial approximation proposed by Fixman [Macromolecules 19, 1204 (1986)] for the square root of the diffusion tensor. We have developed a fast method to continuously determine the validity of the eigenvalue range used in the polynomial approximation, and demonstrated how this range may be quickly updated when necessary. We have also developed a weak first order semiimplicit time integration scheme which offers increased stability in the presence of steep excluded volume potentials. The full algorithm scales roughly as $O\left(N^{2.25}\right)$ and offers substantial computational savings over the standard Cholesky decomposition. The above algorithm was used to obtain scaling exponents for various static and zero shear rate dynamical properties, which are found to be consistent with theoretical and/or experimental predictions. (C) 2000 American Institute of Physics. [S0021-9606(00)51431-7]
\end{abstract}

\section{INTRODUCTION}

The inclusion of hydrodynamic interactions in the simulation of dilute polymer solutions is necessary if one wishes to capture the basic physics of nonfree-draining polymer molecules. ${ }^{1}$ Hydrodynamic interactions have been studied extensively by analytical methods, ${ }^{2-37}$ stochastic simulations, ${ }^{1,38-46}$ and to some extent, by numerical solution of the probability distribution function. ${ }^{47-49}$ There has been recent interest in the rheological and conformational properties of dilute solutions of DNA and other proteins. ${ }^{50-55}$ The treatment of hydrodynamic interactions in these systems, particularly in microfabricated geometries ${ }^{53}$ and near walls, is essential if one wishes to understand their microstructural behavior and subsequently predict their transport properties.

Stochastic simulations of polymers in a dilute solution with fluctuating (i.e., not preaveraged) hydrodynamic interactions have been limited to relatively short chains. ${ }^{56}$ Studies of the behavior of these systems in the long-chain limit have been hindered due to the computational demands associated with the calculation of the hydrodynamic interactions. Such interactions involve the decomposition of the diffusion tensor that appears in the stochastic form of the Fokker-Planck equation. In 1986, Fixman ${ }^{57}$ presented a polynomial approximation to this decomposition which considerably reduced the required computations. At that time, long chains were still out of range, even using Fixman's method. Even with the introduction of fast, inexpensive workstations, Fixman's method has never been applied to long chain polymers, probably due to the lack of an effective means of assuring that polymer configurations remain within the valid range of the approximation.

It is imperative that fast, practical methods of accurately dealing with hydrodynamic interactions be developed if realistic simulations of long chain polymer solutions are to be realized. This is particularly true if one wishes to include fluctuating hydrodynamic interactions in coupled microscopic/continuum calculation schemes, such as the Brownian configuration fields $(\mathrm{BCF})^{58}$ or operator-splitting cumulant-matching $(\mathrm{OSCM})^{59,60}$ methods. In this work we propose one such method.

The outline of this article is as follows. In Sec. II, we present the governing equations, polymer model, and our choice of parameters. In Sec. III, we discuss the Chebyshev polynomial expansion and present a rapid method of assessing the validity of the polynomial expansion. In Sec. IV, a semiimplicit Euler scheme is presented for time integration of the stochastic differential equations. The scaling properties of interest are defined in Sec. V. Details of the simulation are given in Sec. VI; the results are presented and discussed in Sec. VII.

\section{MODEL}

In a Lagrangian frame of reference, the dimensionless ${ }^{61}$ governing equation for the probability distribution function of an isolated "bead-spring" polymer chain in the presence of a velocity gradient $\nabla \mathbf{v}$, can be expressed as ${ }^{4,62,63}$

$$
\frac{\partial \Psi}{\partial t}=-\frac{\partial}{\partial \mathbf{R}} \cdot[(\mathbf{K} \cdot \mathbf{R}+\mathbf{D} \cdot \mathbf{F}) \Psi]+\frac{\partial}{\partial \mathbf{R}} \cdot \mathbf{D} \cdot \frac{\partial}{\partial \mathbf{R}} \Psi .
$$

Here $N$ is the number of beads in the chain, $\mathbf{R}$ is a vector containing the $3 N$ spatial coordinates of the beads that constitute the polymer chain, $\mathbf{D}$ is a $3 N \times 3 N$ diffusion tensor, $\mathbf{F}$ is a force vector of length $3 N$ acting on the chain. The $3 N$ $\times 3 N$ matrix $\mathbf{K}$ is block diagonal with diagonal components $(\nabla \mathbf{v})^{T}$.

Equation (1) can be expressed as a stochastic differential equation in the $3 N$ spatial coordinates:

$$
\begin{aligned}
& d \mathbf{R}=[\mathbf{K} \cdot \mathbf{R}+\mathbf{D} \cdot \mathbf{F}] d t+\sqrt{2} \mathbf{B} \cdot d \mathbf{w}, \\
& \mathbf{D}=\mathbf{B} \cdot \mathbf{B}^{T} .
\end{aligned}
$$


The components $d \mathbf{w}$ are obtained from a real-valued Gaussian distribution with mean zero and variance $d t$.

Hydrodynamic interactions enter Eq. (2) through the diffusion tensor. In this work we take $\mathbf{D}$ to be the RotnePrager-Yamakawa (RPY) tensor, ${ }^{64}$ which, in dimensionless form, is

$$
\begin{aligned}
& \mathbf{D}_{i i}=\frac{1}{4} \mathbf{I} \\
& \mathbf{D}_{i j}=\frac{3 a}{16 R_{i j}}\left[C_{1} \mathbf{I}+C_{2} \frac{\mathbf{R}_{i j} \mathbf{R}_{i j}}{R_{i j}^{2}}\right] \text { if } i \neq j \quad \text { and } R_{i j} \geqslant 2 a
\end{aligned}
$$$$
\begin{gathered}
\mathbf{D}_{i j}=\frac{1}{4}\left[\left(1-\frac{9 R_{i j}}{32 a}\right) \mathbf{I}+\frac{3}{32} \frac{\mathbf{R}_{i j} \mathbf{R}_{i j}}{a R_{i j}}\right] \\
\text { if } i \neq j \text { and } R_{i j}<2 a,
\end{gathered}
$$

where

$$
\begin{aligned}
& C_{1}=1+\frac{2 a^{2}}{3 R_{i j}^{2}}, \\
& C_{2}=1-\frac{2 a^{2}}{R_{i j}^{2}},
\end{aligned}
$$

and $\mathbf{R}_{i j} \equiv \mathbf{R}_{j}-\mathbf{R}_{i}$. The vector $\mathbf{R}_{i}$ contains the three coordinates of the position vector of the $i$ th bead measured from the center of mass. The RPY tensor is positive-definite for all chain configurations, but exact only as $R_{i j} \rightarrow \infty$. Parameter $a$ represents the dimensionless hydrodynamic radius of each bead, and $\mathbf{I}$ is the $3 \times 3$ identity matrix.

The beads of the polymer chain are connected by finitely extensible nonlinear (FENE) springs, with total potential energy

$$
\phi^{(c)}=\sum_{|i-j|=1}-\frac{1}{2} b \ln \left[1-\frac{R_{i j}^{2}}{b}\right],
$$

where $R_{i j} \equiv \sqrt{\mathbf{R}_{i j} \cdot \mathbf{R}_{i j}}$, and where $b$ is the square of the maximum dimensionless bond length. For excluded volume interactions, we use a soft exponential repulsion with total energy

$$
\phi^{(v)}=\sum_{i<j} A \exp \left[-\alpha R_{i j}\right]
$$

The force appearing in Eqs. (1) and (2) is given by

$$
\mathbf{F}_{\mathbf{i}}=-\frac{\partial}{\partial \mathbf{R}_{i}}\left(\phi^{(c)}+\phi^{(v)}\right) .
$$

As far as scaling laws are concerned, the type or strength of the excluded volume interaction should only serve to shift the point at which long-chain behavior is realized. A soft potential satisfies the purpose of this article, which is to demonstrate the practical application of Fixman's method for long chains, and to generate results for the scaling of static and dynamic chain properties with molecular weight in the presence of fluctuating hydrodynamic interactions. In this article we used $b=56.25, A=75.0$, and $\alpha=4.0$. A cutoff of $R_{i j}=2.5$ was used for $\phi^{(v)}$. The values of $A$ and $\alpha$ are similar to those used by Rey et al. ${ }^{65}$ We used $a=0.4$ for the dimensionless bead hydrodynamic radius.

\section{FIXMAN'S METHOD}

As the length of the polymer chain increases, the calculation of $\mathbf{B}$ from Eq. (3) becomes the computational bottleneck of the stochastic simulation. This operator is not unique; any B satisfying Eq. (3) is valid for the determination of ensemble averages. ${ }^{63}$ Nevertheless, there are two natural choices for $\mathbf{B}$. One is the square-root matrix, which satisfies

$$
\mathbf{D}=\mathbf{S} \cdot \mathbf{S},
$$

with

$$
\mathbf{S}=\mathbf{S}^{T},
$$

for symmetric $\mathbf{D}$. The other arises from the Cholesky decomposition of $\mathbf{D}$, which gives $\mathbf{B}$ as an upper (or lower) triangular matrix. This decomposition scales as $N^{3}$, and we are not aware of faster methods for the calculation of $\mathbf{B} .{ }^{57}$ The exact computation of $\mathbf{S}$, on the other hand, requires solution of an eigenvalue problem, also an $O\left(N^{3}\right)$ process. The Cholesky decomposition is the preferred method in the literature.

Fixman, however, was able to exploit two important facts. First, $\mathbf{S}$ can be approximated and, second, it need not be constructed explicitly. Rather, it is the vector $\mathbf{S} \cdot d \mathbf{w}$ that is required, and computation of its polynomial approximation scales roughly as $N^{2.25}$. For a detailed discussion on the vector approximation, we refer the reader to Fixman's original work. ${ }^{57}$ Here we present the algorithm, and discuss some key issues that arise in the use of Fixman's method.

Let $s^{(d)}$ be the Chebyshev polynomial approximation ${ }^{66}$ of the scalar function $\sqrt{d}$ over the range $\left[\lambda_{\min }, \lambda_{\max }\right]$. Then $s^{(d)}$ can be expressed as

$$
s^{(d)}=\sum_{l=0}^{L} a_{l} C_{l},
$$

where

$$
\begin{aligned}
& C_{0}=1, \\
& C_{1}=d_{a} d+d_{b}, \\
& C_{l+1}=2\left(d_{a} d+d_{b}\right) C_{l}-C_{l-1},
\end{aligned}
$$

(these follow from the recursion relations for the Chebyshev polynomials) with

$$
\begin{aligned}
& d_{a}=\frac{2}{\lambda_{\max }-\lambda_{\min }}, \\
& d_{b}=-\frac{\lambda_{\max }+\lambda_{\min }}{\lambda_{\max }-\lambda_{\min }},
\end{aligned}
$$

and $L$ is the order of the polynomial approximation. For the calculation of the Chebyshev coefficients, $a_{l}$, we refer the reader to Canuto et al. ${ }^{66}$

Using the properties of functions of matrices, ${ }^{67}$ generalization of the above scalar case gives $\mathbf{S}^{(D)}$, the Chebyshev polynomial approximation of the matrix function $\mathbf{D}^{1 / 2}$, 


$$
\mathbf{S}^{(D)}=\sum_{l=0}^{L} a_{l} \mathbf{C}_{l},
$$

where

$$
\begin{aligned}
& \mathbf{C}_{0}=\mathbf{I}, \\
& \mathbf{C}_{1}=d_{a} \mathbf{D}+d_{b} \mathbf{I}, \\
& \mathbf{C}_{l+1}=2\left(d_{a} \mathbf{D}+d_{b} \mathbf{I}\right) \mathbf{C}_{l}-\mathbf{C}_{l-1} .
\end{aligned}
$$

The $a_{l}$ are the same Chebschev coefficients as obtained in the scalar case and the eigenvalues of $\mathbf{D}$ are bounded by $\left[\lambda_{\min }, \lambda_{\max }\right]$. The explicit calculation of $\mathbf{S}^{(D)}$ is not necessary, because the quantity of interest is $\mathbf{S} \cdot d \mathbf{w}$, whose polynomial approximation $\mathbf{y}$ may be obtained by a series of matrixvector multiplications

$$
\begin{aligned}
& \mathbf{y}=\mathbf{S}^{(D)} \cdot d \mathbf{w}=\sum_{l=0}^{L} a_{l} \mathbf{x}_{l}, \\
& \mathbf{x}_{0}=d \mathbf{w}, \\
& \mathbf{x}_{1}=\left[d_{a} \mathbf{D}+d_{b} \mathbf{I}\right] \cdot d \mathbf{w}, \\
& \mathbf{x}_{l+1}=2\left[d_{a} \mathbf{D}+d_{b} \mathbf{I}\right] \cdot \mathbf{x}_{l}-\mathbf{x}_{l-1} .
\end{aligned}
$$

The Chebyshev approximation can be calculated to any desired accuracy, provided $\lambda_{\max }$ and $\lambda_{\min }$ of $\mathbf{D}$ are known. The explicit calculation of these eigenvalues for each configuration, coupled with a Chebyshev polynomial expansion at each configuration, would result in little or no savings in computational time (compared to the exact Cholesky decomposition). Fixman partially circumvented this difficulty by using preaveraged Rotne-Prager hydrodynamic interactions to obtain approximations for the upper and lower limits of the eigenvalues. There is, however, no guarantee that during a simulation the eigenvalues will remain within the preaveraged limits for all configurations. We show now how to efficiently maintain an accurate polynomial approximation.

The Chebyshev expansion is only valid within the chosen eigenvalue range; we have developed a rapid method to evaluate the error which results from a violation of the eigenvalue limits. Using Eqs. (12), (13), and (24) we have

$$
\lim _{L \rightarrow \infty}[\mathbf{y} \cdot \mathbf{y}]=d \mathbf{w} \cdot \mathbf{D} \cdot d \mathbf{w},
$$

assuming that we are using a valid eigenvalue range. We now define a relative error $E_{f}$ according to

$$
E_{f}=\sqrt{\frac{|\mathbf{y} \cdot \mathbf{y}-d \mathbf{w} \cdot \mathbf{D} \cdot d \mathbf{w}|}{d \mathbf{w} \cdot \mathbf{D} \cdot d \mathbf{w}}} .
$$

If the polynomial approximation given by Eq. (24) were exact, $E_{f}$ would be identically zero for any choice of $d \mathbf{w}$ and Eq. (12) would be satisfied. In other words, $E_{f}$ measures the extent to which the fluctuation-dissipation theorem is violated. Strictly speaking, $E_{f}$ should be small for all possible $d \mathbf{w}$. However, the probability that an arbitrary $d \mathbf{w}$ coupled with an erroneous $\mathbf{S}^{(D)}$ will result in small $E_{f}$ is vanishingly small, as we have verified by performing trial simulations using both the Cholesky decomposition and Fixman's method. The two methods showed identical time series for all ensemble averaged properties.
In practice, the polynomial approximation $\mathbf{y}$ and corresponding $d \mathbf{w}$ can be used to calculate $E_{f}$ for each configuration at each time step with negligible computational cost. If $E_{f}$ proves to be unacceptable, a new eigenvalue range can be computed for the given configuration, and this new range used until another violation of $E_{f}$ occurs. In our work, we use the free software package ARPACK, which is capable of rapidly solving for only the upper and lower eigenvalues of D by the Arnoldi method in $O\left(N^{2}\right)$ operations. ${ }^{68}$

A few more words on the polynomial approximation are in order. The number of terms (or collocation points) that must be retained to achieve a desired accuracy depends on the condition number $\left(C_{n}=\lambda_{\max } / \lambda_{\min }\right)$ of $\mathbf{D}$. We found this dependence to scale roughly as $C_{n}^{0.5}$ for accuracy of $O\left(10^{-3}\right)$, which is consistent with the findings of Fixman. ${ }^{57}$ This relatively weak dependence makes the computational savings (over Cholesky factorization) increase with chain length.

Excluded volume potentials facilitate implementation of Fixman's method. Aside from the loss of physical meaning of the RPY hydrodynamic approximation in cases where overlaps between beads are common, it is useful to note that Fixman's method itself becomes impractical in the absence of excluded volume. The RPY tensor achieves positivedefiniteness by ignoring the overlap volume during integration of the viscous dissipation (recall that the RPY tensor is the result of seeking an upper limit to the true viscous dissipation). While ignoring the overlap volume assures us of a positive definite $\mathbf{D}$, it also tends to drive $\lambda_{\text {min }}$ down with increasing overlap. In the limit of complete overlap, $\lambda_{\text {min }}$ approaches zero, and $C_{n}$ tends to infinity. For example, consider a pair of tangent beads oriented along the $x$-axis, and let $C_{n}^{o}$ be the condition number at that orientation. With our choice of the hydrodynamic radius, we find $C_{n} / C_{n}^{o}$ $=(2.23,12.08,24.38)$ for overlaps of $50 \%, 90 \%$, and $95 \%$, respectively. Here, a $0 \%$ overlap corresponds to tangent beads of radius $a$, and $100 \%$ overlap indicates complete superposition of the beads. Recall that the number of collocation points needed to achieve a given accuracy increases with increasing $C_{n}$. Once the number of collocation points used approaches $3 N$, the Cholesky decomposition becomes faster than the polynomial approximation.

\section{TIME INTEGRATION}

We can integrate Eq. (2) over an interval $\Delta t$ to obtain

$$
\begin{aligned}
\mathbf{R}(t+\Delta t)-\mathbf{R}(t)= & \int_{t}^{t+\Delta t}[\mathbf{K} \cdot \mathbf{R}+\mathbf{D} \cdot \mathbf{F}] d t^{\prime} \\
& +\sqrt{2} \int_{\mathbf{w}(t)}^{\mathbf{w}(t+\Delta t)} \mathbf{B} \cdot d \mathbf{w}^{\prime} .
\end{aligned}
$$

Steep gradients in excluded volume and FENE potentials require the use of small time steps when an explicit Euler scheme is used to integrate Eq. (30). We have therefore developed a semiimplicit method that scales as $N^{2}$ and allows stable integration for reasonable time step sizes. Suppose we use an explicit Euler scheme on the second integral in Eq. 
(30), and an implicit Euler scheme on the first integral, but treat $\mathbf{D}(t)$ as constant over the time integration. This semiimplicit method is written as

$$
\begin{aligned}
\mathbf{R}(t+\Delta t)-\mathbf{R}(t)= & {[\mathbf{K}(t+\Delta t) \cdot \mathbf{R}(t+\Delta t)+\mathbf{D}(t) \cdot \mathbf{F}(t} \\
& +\Delta t)] \Delta t+\sqrt{2} \mathbf{B}(t) \cdot \Delta \mathbf{w},
\end{aligned}
$$

and maintains the requirement that Eq. (3) be satisfied at each time step. Note that this is equivalent to using a pure implicit Euler scheme on the right-hand side of Eq. (30), and then ignoring the quantity

$$
\begin{aligned}
E_{i e e}= & {[(\mathbf{D}(t+\Delta t)-\mathbf{D}(t)) \cdot \mathbf{F}(t+\Delta t)] \Delta t+\sqrt{2}[\mathbf{B}(t+\Delta t)} \\
& -\mathbf{B}(t)] \cdot \Delta \mathbf{w},
\end{aligned}
$$

which we expect to be $o(\Delta t)$ as $\Delta t \rightarrow 0$, resulting in a first order scheme. Equation (31) is similar to a semiimplicit scheme proposed by Öttinger ${ }^{63}$ in that portions of the drift term are treated implicitly, while the diffusion term is treated explicitly. Our semiimplicit method, which treats F implicitly, provides the desired increase in stability, and we expect (and confirm below) that weak first-order accuracy is maintained.

Equation (31) represents a system of $3 N$ nonlinear equations, which can be solved by Newton iteration. The key to Newton iteration is the construction of the $3 N \times 3 N$ Jacobian matrix (which in this case can be determined analytically), and subsequent solution of the system to get a correction $\delta \mathbf{R}$. With hydrodynamic interactions, the Jacobian is a full matrix, but with proper preconditioning, the iterative solver GMRES $^{69}$ solves the system rapidly in $O\left(N^{2}\right)$ operations. Thus, the overall scaling of the algorithm remains $O\left(N^{2.25}\right)$.

During the intermediate steps of a Newton iteration, it is possible to sample configurations which contain a bond length greater than $\sqrt{b}$. We therefore define a Hookean-type potential between beads $i$ and $j$ as

$$
\phi_{i j}^{H}=\frac{R_{i j}^{2}}{2\left[1-\left(R_{\max }^{2} / b\right)\right]},
$$

which is used in place of the FENE potential if $R_{i j}>R_{\max }$. Note that this replacement is rarely used, and only for intermediate steps of the Newton iteration. The chain configurations at the end of each time step always lie in the valid domain of the FENE potential. In this paper we used $R_{\max }^{2}$ $=0.97 \mathrm{~b}$.

To test the accuracy of our semiimplicit method, we compared it with the explicit Euler scheme by computing ensemble average properties $\left(R^{2}, R_{g}^{2},\left\langle Q^{2}\right\rangle\right)$ for ensembles of 10000 chains of 10 beads with FENE springs and fluctuating hydrodynamic interactions. The comparisons were conducted both at equilibrium and at a Weissenberg (We) number $^{70}$ of 1.0. The two schemes were found to be consistent, with ensemble averages converging linearly to the same values at zero time step.

The semiimplicit integration scheme resulted in a code that was about ten times faster for a given accuracy than that of the explicit Euler, as well as properties that were some- what smoother in time. Note that the choice of integration scheme is irrelevant in the implementation of Fixman's method. We choose to use the semiimplicit scheme because it allowed us to simulate chain lengths of up to 125 beads on simple workstations without loss of accuracy.

\section{SCALING PROPERTIES}

The physical properties investigated in this article include the diffusion coefficient $D$, the radius of gyration $R_{g}$, the end-to-end distance $R$, the shear viscosity $\eta$, and first normal stress coefficient $\Psi_{1}$.

The radius of gyration and end-to-end distance are given by

$$
R_{g}^{2}=\frac{1}{2 N^{2}} \sum_{i, j}\left\langle R_{i j}^{2}\right\rangle,
$$

and

$$
R^{2}=\left\langle R_{1 N}^{2}\right\rangle \text {. }
$$

The theoretical scaling for these static properties is ${ }^{4}$

$$
R_{g}^{2} \propto R^{2} \propto N^{2 \nu} .
$$

In a good solvent, as $N \rightarrow \infty$, the scaling exponent has a theoretical value of $\nu \cong 0.588$ from a perturbation analysis, and an experimental value of $\nu \cong 0.6 .^{71,72}$

The diffusion coefficient can be calculated from the center-of-mass trajectory

$$
\left\langle\Delta R_{c}^{2}\right\rangle=6 D t .
$$

We also computed the diffusivity by the approximate Kirkwood formula ${ }^{62}$

$$
D^{(\text {Kirkwood })}=\frac{1}{N^{2}} \sum_{i, j} \operatorname{Tr}\left(\left\langle\mathbf{D}_{i j}\right\rangle\right) .
$$

The two results were indistinguishable within the error of the simulation. In the nonfree-draining limit, $D \propto R_{g}^{-1}$, and we have

$$
D \propto N^{-\nu} .
$$

The polymer contribution to the stress tensor can be expressed in dimensionless form as ${ }^{62}$

$$
\boldsymbol{\tau}_{p}=\sum_{i}\left\langle\mathbf{R}_{i} \mathbf{F}_{i}\right\rangle
$$

The steady shear properties of concern in this article are the dimensionless viscosity and first normal stress coefficient defined by ${ }^{62}$

$$
\eta=-\frac{\tau_{12}}{\dot{\gamma}}
$$

and

$$
\Psi_{1}=-\frac{\left(\tau_{11}-\tau_{22}\right)}{\dot{\gamma}^{2}},
$$


TABLE I. Sizes and lengths of simulations. $\lambda_{R}$ refers to the longest nondimensional Rouse relaxation time.

\begin{tabular}{ccc}
\hline \hline No. of beads & Ensemble size & Averaging period $\left(\lambda_{R}\right)$ \\
\hline 20 & 200 & 38 \\
40 & 100 & 38 \\
60 & 50 & 38 \\
80 & 25 & 20 \\
100 & 10 & 15 \\
125 & 14 & 15 \\
\hline \hline
\end{tabular}

where $\dot{\gamma}$ is the nondimensional shear rate. The Zimm model predicts in the limit of zero shear ${ }^{4}$

$$
\eta \propto N^{3 \nu}
$$

and

$$
\Psi_{1} \propto N^{6 \nu} .
$$

\section{SIMULATION DETAILS}

All simulations were equilibrated without hydrodynamic interactions, then restarted with hydrodynamic interactions and again allowed to equilibrate. A dimensionless time step of $\Delta t=0.1$ was used in all cases. This time step is small enough to give good performance of the semiimplicit method (average convergence rate of 2-3 Newton iterations), and trial simulations with various $\Delta t$ show that it gives accurate results. Chains ranging from 20 to 125 beads were simulated at equilibrium and in shear flow at a $\mathrm{We}=1.0$, at which point the transition to zero shear rate dynamic properties is approximately complete. At $\mathrm{We}=1.0$, we found $\left\langle Q^{2}\right\rangle /\left\langle Q^{2}\right\rangle_{\text {eq }}$ $<1.02$ for all chain lengths, where $\left\langle Q^{2}\right\rangle$ is the average square bond length for a given chain. We also found $\left\langle S_{y y}\right\rangle_{\mathrm{eq}} /\left\langle S_{y y}\right\rangle<1.2$ for all chain lengths, where $\left\langle S_{y y}\right\rangle$ is the component of the radius of gyration tensor in the gradient direction. Ensemble sizes and averaging times are given in Table I.

Variance reduction by parallel processes ${ }^{63}$ was used for all simulations, which resulted in improvement over the nonvariance reduced properties, albeit not to the extent that would occur in the absence of excluded volume and nonlinear springs.

\section{RESULTS AND DISCUSSION}

The molecular weight dependence of the physical properties defined in Sec. V is illustrated in Figs. 1, 2, and 3. The slopes of the linear fits and the values of the exponent $\nu$ extracted via the scaling of Sec. IV are presented in Table II. The errors bars on $D$ in Fig. 2 are not shown as they are smaller than the symbols. The error bars on $\eta$ and $\Psi_{1}$ in Fig. 3 are large relative to those of the other properties. This is primarily due to the inefficiency of variance reduction for long chains with excluded volume, and also because of the relatively short simulation time and small ensembles.

The ratio $R^{2} / R_{g}^{2}$ was also calculated and found to be independent of molecular weight. We determined $R^{2} / R_{g}^{2}$ $=6.3 \pm 0.3$, in excellent agreement with the value of $6.2539 \pm .0078$ from recent Monte Carlo simulations reported

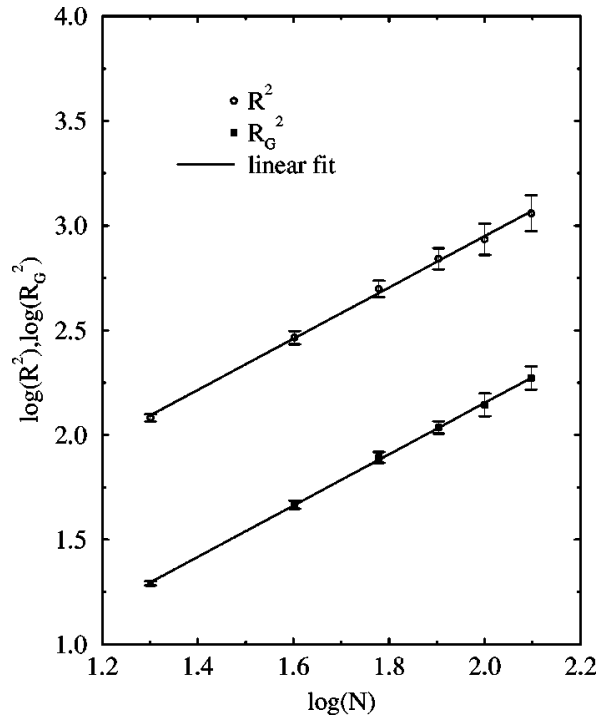

FIG. 1. Molecular weight scaling for $R^{2}$ and $R_{g}^{2}$.

by Li et al. ${ }^{72}$ We also calculated the ratio $R_{h} / R_{g}$, where $R_{h}$ is the effective chain hydrodynamic radius from the StokesEinstein equation for the diffusivity

$$
D=\frac{k T}{6 \pi \eta_{s} R_{h}} .
$$

In dimensionless form, $R_{h}$ is given by

$$
R_{h}=\frac{a}{4 D} .
$$

We found $R_{h} / R_{g}=0.62 \pm 0.03$ for all molecular weights. This compares favorably to the theoretical value of 0.64 (from renormalization group theory), ${ }^{73}$ but less favorably to the value of $\sim 0.67$ from the Kirkwood-Riseman theory. ${ }^{2}$ The latter prediction relies on preaveraging of the hydrodynamic interaction tensor. The renormalization group calculation does not make this approximation. Both of these theories assume $N \rightarrow \infty$.

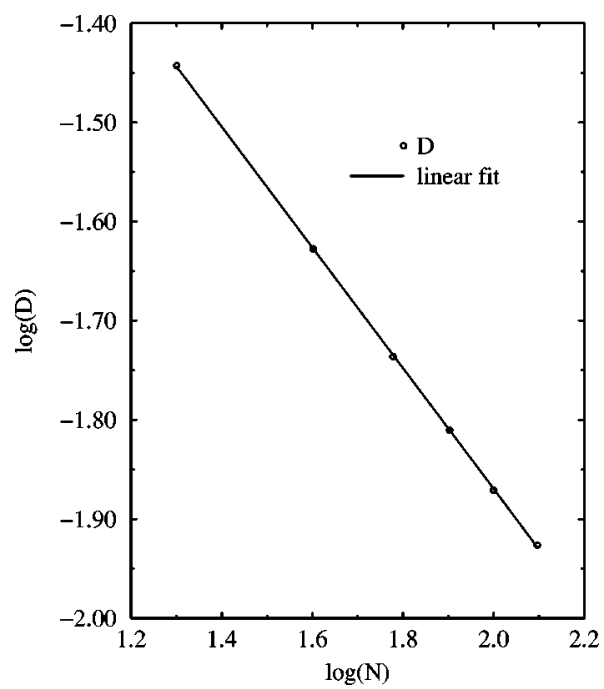

FIG. 2. Molecular weight scaling for $D$. 


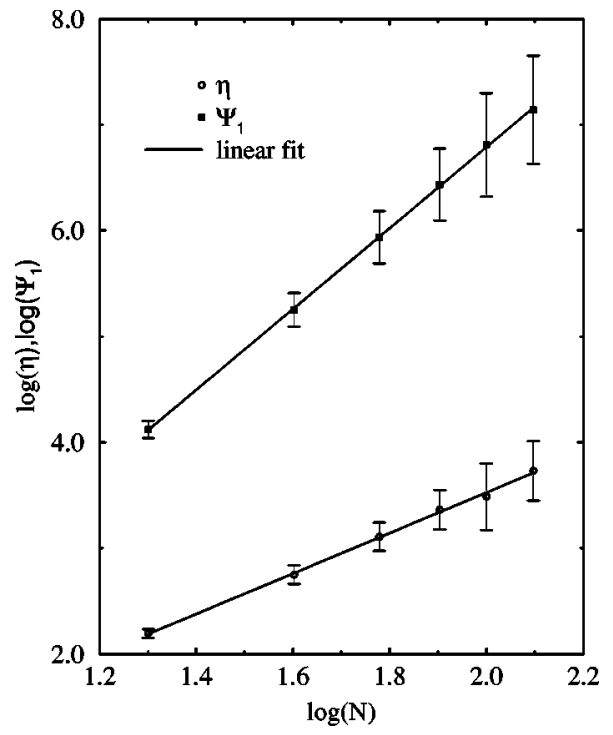

FIG. 3. Molecular weight scaling for $\eta$ and $\Psi_{1}$.

The scaling exponent for the static properties $R^{2}$ and $R_{g}^{2}$ is $\sim 4 \%$ higher than the theoretical limit of $\nu=0.588$, and $\sim 2 \%$ higher than the accepted experimental value of $0.6 .^{71}$ The scaling exponent for $D$ is closer to the predicted values but still slightly high. The calculated scaling exponents for $D$ and $R_{g}^{2}$ agree to within $0.7 \%$, indicating that the nonfree draining assumption is realized for our choice of parameters. The exponents derived for the dynamic properties $\eta$ and $\Psi_{1}$ are nearly indistinguishable and overpredict experimental and theoretical values by $6.3 \%$ and $8.5 \%$, respectively.

The predicted scaling exponent in all cases lies above the theoretical limit of $\nu=0.588$. This is consistent with the Monte Carlo simulations of $\mathrm{Li}$ et al. ${ }^{72}$ which show that the effective scaling exponents corresponding to $R^{2}$ and $R_{g}^{2}$ approach the theoretical value from above. We attribute part of these discrepancies to the fact that we have not reached the infinite chain length limit in our simulations.

Table II demonstrates the internal consistency of our simulations, and in all cases agreement to within $\sim 6 \%$ of experimental values provides an external validation of both our implementation of Fixman's method and our semiimplicit integration scheme.

These simulations, all of which were performed on simple workstations, demonstrate the ease with which Fixman's method can be applied to stochastic processes. The method presents a considerable savings in computational time over the standard Cholesky factorization, and the semiimplicit time integration scheme further improves efficiency

TABLE II. Summary of values of slopes and corresponding $\nu$ as obtained from various physical quantities.

\begin{tabular}{crc}
\hline \hline Physical Quantity & Slope & $\nu$ \\
\hline$R^{2}$ & 1.224 & 0.612 \\
$R_{g}^{2}$ & 1.226 & 0.613 \\
$D_{c}$ & -0.609 & 0.609 \\
$\eta$ & 1.913 & 0.638 \\
$\Psi_{1}$ & 3.825 & 0.637 \\
\hline \hline
\end{tabular}

by about a factor of ten. More importantly, it offers the opportunity to include fluctuating hydrodynamic interactions in studies of the dynamics of long polymers in complex flows.

Note added in proof. Since the submission of this paper we have become aware of recent work in the area of hydrodynamic interactions in linear polymer systems. Lyulin et al. [A. V. Lyulin, D. B. Adolf, and G. R. Davies, J. Chem. Phys. 111, 758 (1999)] performed bead-rod simulations with excluded volume and RPY HI. they do not appear to have used Fixman's method. Rzehak et al. [R. Ryzhak, W. Kromen, T. Kawakatsu, and W. Zimmerman, Eur. Phys. J E 2, 3 (2000)] applied Fixman's method to bead-spring simulations on individual tethered chains of up to 200 beads in uniform flow including FENE, excluded volume, and HI effects. These authors included an excluded volume with all applications of Fixman's method, and checked the validity of the eigenvalue range by periodically diagonalizing the diffusion tensor (an $N^{3}$ operation). These authors used the Oseen (not RPY) HI tensor, and the inclusion of excluded volume was necessary to ensure that the Oseen tensor remained positive-definite. For this system, they found that the eigenvalue approximations proposed by Fixman ${ }^{57}$ appeared to be valid. Kröger et al. [M. Kröger, A. Alba, M. Laso, and H. C. Öttinger (unpublished)] applied Fixman's method to Hookean chains with RPY HI in the absence of excluded volume. They used the eigenvalue approximations proposed by Fixman. ${ }^{57}$

\section{ACKNOWLEDGMENTS}

Acknowledgment of support is made to the National Science Foundation (CTS-9901430 and CTS-9815518) and to the donors of the Petroleum Research Fund, administered by the American Chemical Society. Many thanks to K. Arun Kumar, William B. Black, and Hans-Christian Öttinger for helpful discussions.

${ }^{1}$ D. Petera and M. Muthukumar, J. Chem. Phys. 111, 7614 (1999).

${ }^{2}$ J. G. Kirkwood and J. Riseman, J. Chem. Phys. 16, 565 (1948).

${ }^{3}$ B. H. Zimm, J. Chem. Phys. 24, 269 (1956).

${ }^{4}$ M. Doi and S. F. Edwards, The Theory of Polymer Dynamics (Clarendon, Oxford, 1986).

${ }^{5}$ M. Fixman, J. Chem. Phys. 42, 3831 (1965).

${ }^{6}$ C. W. Pyun and M. Fixman, J. Chem. Phys. 42, 3838 (1965).

${ }^{7}$ C. W. Pyun and M. Fixman, J. Chem. Phys. 44, 2107 (1966).

${ }^{8}$ M. Fixman, J. Chem. Phys. 45, 785 (1966).

${ }^{9}$ M. Fixman, J. Chem. Phys. 45, 793 (1966).

${ }^{10}$ H.-C. Öttinger, J. Chem. Phys. 86, 3731 (1987).

${ }^{11}$ H.-C. Ottinger, J. Chem. Phys. 90, 463 (1989).

${ }^{12}$ J. J. Magda, R. G. Larson, and M. E. Mackay, J. Chem. Phys. 89, 2504 (1988).

${ }^{13}$ R. G. Larson and J. J. Magda, Macromolecules 22, 3004 (1989).

${ }^{14}$ W. Zykla and H.-C. Öttinger, J. Chem. Phys. 90, 474 (1989).

${ }^{15}$ H.-C. Öttinger, J. Non-Newtonian Fluid Mech. 26, 207 (1987).

${ }^{16}$ L. E. Wedgewood and H.-C. Öttinger, J. Non-Newtonian Fluid Mech. 27, 245 (1988).

${ }^{17}$ L. E. Wedgewood, D. N. Ostrov, and R. B. Bird, J. Non-Newtonian Fluid Mech. 40, 119 (1991).

${ }^{18}$ Y. Oono, Adv. Chem. Phys. 61, 301 (1985).

${ }^{19}$ Y. Oono and M. Kohmoto, J. Chem. Phys. 78, 520 (1983).

${ }^{20}$ Y. Oono, J. Chem. Phys. 79, 4629 (1983).

${ }^{21}$ B. Schaub, B. A. Friedman, and Y. Oono, Phys. Lett. A 110, 136 (1985).

${ }^{22}$ A. Jagannathan, B. Schaub, and Y. Oono, Phys. Lett. A 113, 341 (1985).

${ }^{23}$ A. Jagannathan, Y. Oono, and B. Schaub, J. Chem. Phys. 86, 2276 (1987).

${ }^{24}$ B. Schaub, D. B. Creamer, and H. Johannesson, J. Phys. A 21, 1431 (1988)

${ }^{25}$ S. Wang, Phys. Rev. A 40, 2137 (1989). 
${ }^{26}$ S. Wang, J. Chem. Phys. 92, 7618 (1990).

${ }^{27}$ H.-C. Öttinger, J. Non-Newtonian Fluid Mech. 33, 53 (1989).

${ }^{28}$ H.-C. Öttinger, Phys. Rev. A 40, 2664 (1989).

${ }^{29}$ H.-C. Ottinger, Phys. Rev. A 41, 4413 (1990).

${ }^{30} \mathrm{~W}$. Zykla and H.-C. Öttinger, Macromolecules 24, 484 (1991)

${ }^{31}$ P. R. Baldwin and E. Helfand, Phys. Rev. A 41, 6772 (1990).

${ }^{32}$ R. S. Adler, J. Chem. Phys. 69, 2849 (1978).

${ }^{33}$ S. Q. Wang and K. F. Freed, J. Chem. Phys. 88, 3944 (1988).

${ }^{34}$ Y. Rabin, Europhys. Lett. 7, 25 (1988).

${ }^{35}$ Y. Rabin, S. Q. Wang, and K. F. Freed, Macromolecules 22, 2420 (1989).

${ }^{36}$ Y. Rabin, S. Q. Wang, and D. B. Creamer, J. Chem. Phys. 90, 570 (1989).

${ }^{37}$ H. P. Wittmann and G. H. Fredrickson, J. Phys. I 4, 1791 (1994).

${ }^{38}$ B. H. A. A. van den Brule, J. Non-Newtonian Fluid Mech. 47, 357 (1993).

${ }^{39}$ J. J. L. Cascales, F. G. Diaz, and J. G. de la Torre, Polymer 36, 345 (1995).

${ }^{40}$ M. Herrchen and H.-C. Öttinger, J. Non-Newtonian Fluid Mech. 17, 68 (1997).

${ }^{41}$ M. Fixman, J. Chem. Phys. 69, 1527 (1978).

${ }^{42}$ M. Fixman, Macromolecules 14, 1710 (1981).

${ }^{43}$ D. L. Ermak and J. A. McCammon, J. Chem. Phys. 69, 1352 (1978).

${ }^{44}$ S. A. Allison and J. A. McCammon, Biopolymers 23, 167 (1984).

${ }^{45}$ C. Pierleoni and J. P. Ryckaert, Macromolecules 28, 5097 (1995).

${ }^{46}$ Z. Xu, S. Kim, and J. J. de Pablo, J. Chem. Phys. 101, 5293 (1994).

${ }^{47}$ W. S. Stewart and J. P. Sorensen, Trans. Soc. Rheol. 16, 1 (1972).

${ }^{48}$ X. Fan, J. Chem. Phys. 85, 6237 (1985).

${ }^{49}$ X. Fan, J. Non-Newtonian Fluid Mech. 17, 125 (1985).

${ }^{50}$ T. C. B. Kwan and E. S. G. Shaqfeh, J. Non-Newtonian Fluid Mech. 82, 139 (1999).

${ }^{51}$ P. G. de Gennes, Proc. Natl. Acad. Sci. USA 96, 7262 (1999).

${ }^{52}$ B. H. Zimm, Macromolecules 31, 6089 (1998).

${ }^{53}$ B. B. Haab and R. A. Mathies, Anal. Chem. 71, 5137 (1999).

${ }^{54}$ R. G. Larson, T. T. Perkins, D. E. Smith, and S. Chu, Phys. Rev. E 55, 1794 (1997)

${ }^{55}$ R. G. Larson, H. Hua, D. E. Smith, and S. Chu, J. Rheol. 43, 267 (1999).

${ }^{56}$ J. G. H. Cifre and J. G. de la Torre, J. Rheol. 43, 339 (1999).
${ }^{57}$ M. Fixman, Macromolecules 19, 1204 (1986).

${ }^{58}$ J.-C. Öttinger, B. H. A. A. van der Brule, and M. A. Hulsen, J. NonNewtonian Fluid Mech. 70, 255 (1997).

${ }^{59}$ T. W. Bell, Ph.D. thesis, University of Wisconsin-Madison, 1998.

${ }^{60}$ R. M. Jendrejack, T. W. Bell, J. J. de Pablo, and M. D. Graham, Proceedings of the XIIIth International Congress on Rheology, Cambridge, UK, 2000 (unpublished).

${ }^{61}$ The quantities used for nondimensionalization throughout this article are $\sqrt{k T / H}$ for distance, $\zeta / 4 H$ for time, $k T$ for energy, $\sqrt{k T H}$ for force, and $n k T$ for the stress tensor. The parameter $k$ is Boltzmann's constant, $T$ is temperature, $H$ is Hooke's spring constant, $n$ is the number density of polymer, and $\zeta$ is the bead friction coefficient.

${ }^{62}$ R. B. Bird, C. F. Curtiss, R. C. Armstrong, and O. Hassager, Dynamics of Polymeric Liquids (Wiley, New York, 1987), Vol. 2.

${ }^{63}$ H.-C. Öttinger, Stochastic Processes in Polymeric Fluids (Springer, Berlin, 1996).

${ }^{64}$ J. Rotne and S. Prager, J. Chem. Phys. 50, 4831 (1969).

${ }^{65}$ A. Rey, J. Freire, and J. G. de la Torre, Macromolecules 24, 4666 (1991).

${ }^{66}$ C. Canuto, M. Y. Hussaini, A. Quarteroni, and T. A. Zang, Spectral Methods in Fluid Dynamics (Springer-Verlag, Berlin, 1988).

${ }^{67}$ C. R. Wylie and L. C. Barrett, Advanced Engineering Mathematics, 6th ed. (McGraw-Hill, New York, 1995).

${ }^{68}$ R. B. Lehoucq, D. C. Sorensen, and C. Yang, Arpack Users Guide: Solution of Large Scale Eigenvalue Problems by Implicitly Restarted Arnoldi Methods, 1997, ftp://ftp.caam.rice.edu/pub/software/ARPACK.

${ }^{69}$ Y. Saad and M. H. Schultz, SIAM (Soc. Ind. Appl. Math.) J. Sci. Stat. Comput. 7, 856 (1986).

${ }^{70}$ In our nondimensionalization, the Weissenberg number is defined as We $=\dot{\gamma} \tau_{1}$, where $\tau_{1}=2 / \sin (\pi / 2 N)$ is the longest Rouse relaxation time, and $\dot{\gamma}$ is the shear rate (nondimensionalized with $\zeta / 4 H$ ).

${ }^{71}$ G. R. Strobl, The Physics of Polymers (Springer, Berlin, 1997).

${ }^{72}$ B. Li, N. Madras, and A. Sokal, J. Stat. Phys. 80, 661 (1995).

${ }^{73}$ Y. Oono and M. Kohmoto, J. Chem. Phys. 78, 520 (1983). 